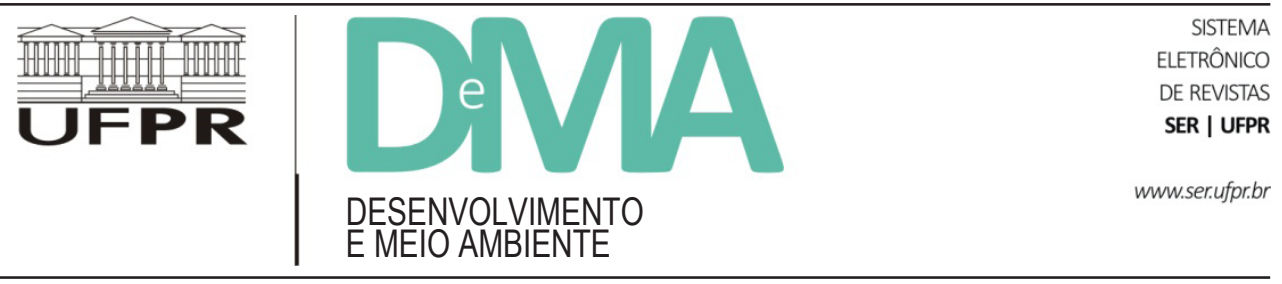

\title{
Replanter une forêt en société postcoloniale: conservations ordinaires et participatives à l'Ile de La Réunion
}

\author{
Replantar uma floresta numa sociedade pós-colonial: conservações \\ regulares e participativas na I/ha Reunião
}

\section{Replanting a Forest in Post-Colonial Society: Ordinary and Participatory Conservations at Reunion Island}

\author{
Marie THIANN-BO MOREL ${ }^{1 *}$ \\ ${ }^{1}$ Université de La Réunion, Le Tampon, La Réunion. \\ *Contact: marie.thiannbo@univ-reunion.fr
}

Article reçu le 29 février 2016, version finale acceptée le 4 juillet 2016.

RÉSUMÉ: Au cours d'une enquête ethnographique, nous avons pu étudier un projet de développement durable et participatif initié par des personnels du Parc National de La Réunion. Ce projet vise la création de Pépinières d'Espèces Indigènes de la Réunion (PEIRun). S'attacher à en comprendre l'élaboration et la mise en œuvre révèle d'une part les stratégies d'empowerment des acteurs - professionnels ou ordinaires - impliqués dans ce projet et d'autre part comment les pratiques idoines de « conservation » se répondent et s'élèvent contre l'hégémonie perçue d'une forme unique de préservation de la biodiversité. Ce projet opère en effet sur un terrain - La Réunion, dont la spécificité réside dans un contexte postcolonial qui favorise des rapports de domination entre les acteurs qu'ils soient directement ou symboliquement impliqués dans ce projet. Après avoir exposé comment la grille de lecture du post-colonialisme permettait de comprendre les sentiments de justice et d'injustice environnementales des acteurs, nous analyserons dans quelles mesures ces justices environnementales seraient conciliables. Un passage par l'historique de la construction du Parc National de La Réunion est alors nécessaire pour cerner les rapports de force qui s'y jouent. Nous verrons alors que la participation devient un enjeu de justice environnementale au niveau local. En nous intéressant aux stratégies d'empowerment des acteurs impliqués dans le projet, nous étudierons comment les porteurs du projet PEIRun cherchent à réparer les injustices qu'ils perçoivent dans leur pratiques professionnelles et comment les populations ciblées par leur projet répondent ou non à cet objectif avec leurs propres attentes.

Mots-clés: Ile de La Réunion; invasion biologique; participation et empowerment; postcolonialisme. 
RESUMO: Durante uma pesquisa etnográfica, estudamos um projeto de desenvolvimento sustentável e participativo iniciado pela equipe do Parque Nacional de Reunião. Este projeto envolve a criação de Viveiros de Espécies Nativas de Reunião (PEIRun). Procurar compreender o seu desenvolvimento e a sua implementação revela, de um lado, as estratégias de empoderamento dos atores - profissionais ou pessoas comuns - envolvidos neste projeto; de outro lado, revela como as práticas adequadas de "conservação" respondem e se levantam contra a perspectiva hegemônica, percebida como a forma única de conservação da biodiversidade. Este projeto se desenvolve em um espaço específico - a Reunião, cuja especificidade reside no seu contexto pós-colonial que favorece as relações de dominação entre os atores de forma direta ou simbolicamente envolvidos neste projeto. Depois de explicar como a perspectiva de leitura do pós-colonialismo permitia compreender os sentimentos de justiça e injustiça ambiental dos atores, analisamos em que medida estas justiças ambientais seriam conciliáveis. Uma retrospectiva da história da construção do Parque Nacional da Reunião é, então, necessária para identificar as relações de poder que ali operam. Veremos desta forma que a participação se torna um embate sobre a justiça ambiental em nível local. Ao nos interessarmos pelas estratégias de empoderamento dos atores envolvidos no projeto, estudamos como os responsáveis pelo projeto PEIRun buscam corrigir as injustiças que percebem em suas práticas profissionais e como as populações alvo do seu projeto respondem ou não a esse objetivo com as suas próprias expectativas.

Palavras-chave: Ilha da Reunião; invasão biólogica; participação e empoderamento; pós-colonialismo.

ABSTRACT: During an ethnographic study, we studied a project of sustainable and participatory development initiated by members of the National Park of La Reunion. This project involves the creation of Nurseries of Indigenous Species (PEIRun). Seek to understand the development and implementation of this project reveals firstly the empowerment strategies of the stakeholders - professional or ordinary - involved in this project and also how each way of nature conservation meets and rises up against what is perceived as an hegemonic form of biodiversity conservation. The project lies in an island - Reunion, whose specificity lies in a postcolonial context that promotes relations of domination between stakeholders. After explaining how the postcolonial reading grid leads to understand the feelings of injustice of the stakeholders, we will analyze to what extent these environmental justices would reconcile. We will see that participation becomes an issue of environmental justice at the local level. As we interested in empowerment strategies of the stakeholders involved in the project, we will explore how the project managers are seeking to redress the injustices they perceive in their professional practices and how the population meets or does not meet this aim with their own expectations

Keywords: Réunion Island; invasive specie; participation \& empowerment; postcolonialism.

\section{Introduction}

\subsection{Le projet PEI-Run}

Les Pépinières d'Espèces Indigènes de la Réunion (PEI-Run) sont le résultat d'un projet de développement durable et participatif mis en place et piloté par des personnels du Parc National de La Réunion (PNR). Ce projet affiche un double objectif de conservation impliquant aussi bien patrimoine naturel que culturel. D'une part, il cherche à réduire un risque majeur pour la biodiversité locale, les invasions biologiques, terme désignant « la prolifération d'espèces animales ou végétales, généralement exotiques, qui réduit la biodiversité en occupant les niches écologiques des espèces locales » (Claeys $\&$ Sirost, 2010, p. 9). Les pépinières vont constituer des espaces de connexions entre les milieux naturels fragmentés par ces invasions (Baret et al., 2012). D'autre part, le projet vise la mise en place de pratiques durables par les populations riveraines des sites envahis et des pépinières. Pour atteindre ces objectifs, l'implication et la participation des 
populations locales sont indispensables (e.g. Baret et al., 2013). Les porteurs du projet souhaitent ainsi laisser aux associatifs sélectionnés la liberté de s'approprier le projet et de le conduire en autonomie sur le long terme.

Le projet repose sur deux croyances. D'abord, il suppose qu'il existe une tradition réunionnaise aux pratiques environnementales vertueuses. C'est cette tradition orale qu'il s'agit de sauver de l'oubli. Une étape du projet attribuée au sociologue consiste donc à recueillir cette mémoire où serait captif un capital de bonnes pratiques du tan lontan (d'autrefois). Le projet en appelle aussi à une participation citoyenne sur des territoires considérés a priori comme désertés physiquement et symboliquement par les riverains. La deuxième croyance postule que l'envahissement des sites forestiers provient d'un abandon collectif par les riverains. Le bien commun « forêt à protéger » serait à (re)construire de toute pièce. Le projet possède sa propre cohérence patrimoniale sans présumer l'existence d'autres formes de patrimonialisation. Les porteurs du projet considèrent ainsi qu'ils militent pour la reconnaissance d'une dimension anthropocentrée de la conservation au sein du parc.

Sur le papier, le projet semble vouloir appliquer une logique conservationniste au patrimoine culturel. Tout comme les espèces biologiques seraient menacées par une trop grande pression anthropique, les savoirs ordinaires sur la nature, fruits d'une culture orale, sont considérés comme en voie de disparition, faute d'avoir été correctement conservés. Dès lors, il convient d'en effectuer la collecte et de les restituer à la génération d'autochtones qui ignorerait tout de cette culture en héritage. La protection du patrimoine culturel relève ici d'une tentative muséographique : le projet consignerait les comportements durables, et suivant une logique patrimoniale, verserait au pot commun un guide des bonnes pratiques à destination de populations dont on construirait le concernement. Les pépinières constitueraient les lieux physiques de la transmission d'une culture « durable » de la nature.

Présenté ainsi, on imagine déjà les écueils que ce type de projet pourrait rencontrer. En affichant la nécessité de revenir à des pratiques ancestrales jugées vertueuses, le projet présume que la (re) découverte du patrimoine suffirait à construire la forêt comme un bien commun. En cela, il sous-estime le poids des injustices perçues, du fatalisme et des rancunes, mais surtout il néglige le sens des pratiques de la nature encore bien installées. Il fait abstraction des formes populaires et vivaces de ces pratiques qui s'épanouissent parfois dans la ruse et l'illégalité revendiquée. Nous verrons que des formes de conservation ordinaire s'expriment déjà là où les participations sont demandées par l'institution porteuse du projet. Ces dernières s'appuient en partie sur une critique du capitalisme ou de la modernité (jugés tous deux comme surexploitant la forêt). Ces critiques, dont nous étudierions les rhétoriques, dénoncent les préjugés des « conservateurs légitimes » qui pointent injustement les populations locales comme forcément et uniformément responsables et fautives des dégradations de leurs milieux. En souhaitant initier une dimension patrimoniale aux dépens de celle qui existe déjà, la promotion d'une tradition posée a priori comme patrimoine vertueux entre alors en décalage avec le vécu des populations.

Ainsi, nous verrons que chaque groupe d'acteurs touché de près ou de loin par le projet développe sa propre éthique et justifie ses pratiques de la nature au regard d'une grille de lecture spécifique des inégalités environnementales qu'il pense subir. Chaque groupe développe ses propres stratégies de transformation sociale. Comme fil rouge, nous nous proposons de suivre les multiples expressions des 
sentiments d'injustice qui permettent d'identifier ces éthiques.

Nous souhaitons ainsi étudier comment les participations des uns et des autres sont influencées par les sentiments d'injustices qu'ils expriment. Nous verrons alors comment d'un côté les porteurs du projet $P E I R u n$ cherchent à réparer les injustices perçues et comment de l'autre, les populations ciblées par le projet répondent ou non à cet objectif avec leurs propres attentes.

Dans un premier temps, nous essaierons de comprendre la demande de participation et les valeurs qui la portent. Nous verrons alors que le projet développe une éthique centrée sur la volonté de réparer des inégalités environnementales (pouvoir d'accès à et sur la ressource) perçues comme héritées de la société de plantation ${ }^{1}$. Or, face à la reproduction des modèles coloniaux de domination, toute tentative de réconciliation entre un pouvoir central (représenté par le PNR) et la population passe par l'étape obligée de la reconnaissance des autochtones. Alors que leur expérience et leur intuition les pousse à minimiser le plus possible sur le terrain la présence institutionnelle du parc pour mieux faire participer ces autochtones, les porteurs $\mathrm{du}$ projet ne se doutent pas que les populations locales développent déjà des pratiques ordinaires de conservation des espaces naturels.

Nous verrons donc, dans un deuxième temps, quelles sont ces logiques à l'œuvre sur les territoires visés par le projet et quelles potentialités conflictuelles elles possèdent vis-à-vis de l'exigence de participation requise par le projet.
Dans un troisième temps, nous répondrons à la problématique en envisageant les freins à la participation. Nous proposerons notamment la grille de lecture du postcolonialisme pour mieux comprendre les rapports de domination.

Nous verrons que l'absence de participation citoyenne n'est pas toujours synonyme d'absence de dynamique de transformation sociale plus juste. Les stratégies d'empowerment des acteurs, si elles n'aboutissent pas à un contexte d'égale prise de parole citoyenne peuvent permettre des formes d'émancipation.

\subsection{Le Parc National de La Réunion}

Le Parc National de La Réunion (PNR) est le neuvième parc national français. Il se compose de deux territoires: un coeur de parc qui couvre $42 \%$ du territoire insulaire disposant d'une réglementation spécifique et une aire d'adhésion qui « résulte de la libre adhésion à la charte du parc national des communes situées à l'intérieur d'un périmètre ${ }^{2} »$; ce dernier représente $35 \%$ de l'île. Au total, le territoire insulaire concerné par le PNR est de 77\%. Créé en 2007, il est le fruit d'une réflexion collective autour d'un objectif initial « magnifier les Hauts ${ }^{3}$ de l'île » (Babou, 2015, p. 12). La construction de ce parc serait le fait d'une élite locale universitaire sollicitant ses réseaux professionnels métropolitains et d'une élite politique qui se sont vus tous deux progressivement dépossédés de la construction des objets patrimoniaux (Babou, 2015). En effet, les

\footnotetext{
1 Se dit d'une société où une seule activité économique destinée à l'exportation (généralement la canne à sucre) est développée avec une politique esclavagiste ou coloniale au détriment d'une agriculture de subsistance (Benoist, 1968).

2 Source: http://www.developpement-durable.gouv.fr

3 Les « hauts » de l'île désignent les espaces situés en altitude, l'espace insulaire est duel et s'organise entre les hauts (ruraux et parfois très escarpés) et les bas (zone littorale) densément peuplés.
} 


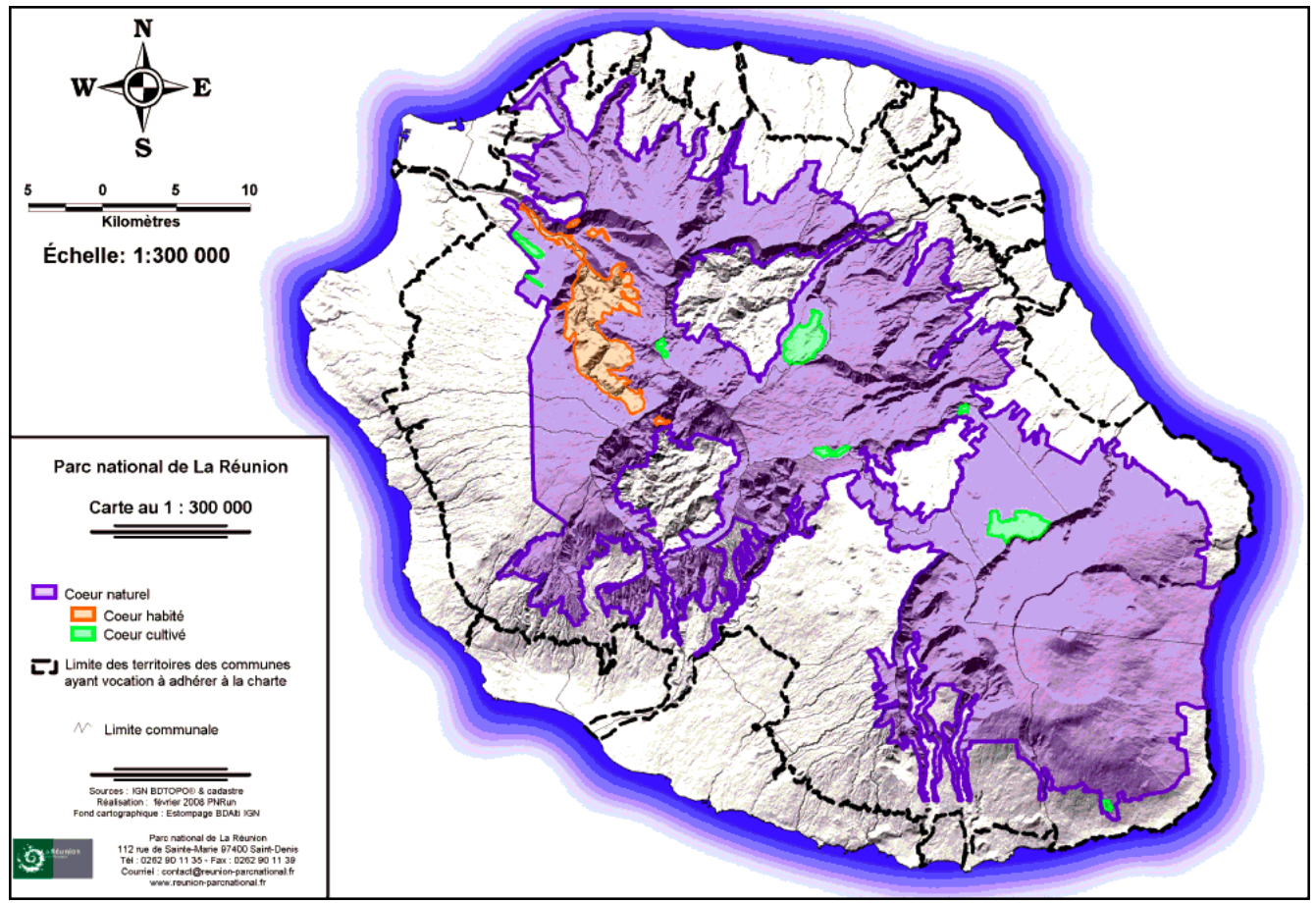

FIGURE 1 - Carte du Parc National de La Réunion.

jeux d'échelle nationaux et internationaux requis par l'inscription au patrimoine mondial de l'UNES$\mathrm{CO}$ ont demandé de s'aligner sur un cahier des charges international hypernormatif. La destination initiale du projet de parc national (portant principalement sur les paysages et les pratiques culturelles des gens des hauts) a progressivement changé vers une «dimension exclusivement naturelle induite par l'UNESCO » (Babou, 2015, p.14). Durant tout ce processus, la population est cantonnée au rôle de destinataire des informations diffusées par les documents de communication, « elle n'est pas associée à ces espaces de discussion » (Babou, 2015, p.18).

La création du Parc apparait donc comme une construction patrimoniale mise en tension entre global et local ; le global s'articulant lui-même entre le national (gestionnaire de l'institution Parc) et l'international (UNESCO). Des tensions en local semblent alors inhérentes au choix de l'outil gestionnaire «parc national ». En effet, cet instrument de politique publique possèderait un aspect descendant (imposition d'une réglementation) et dual (entre le national et le territorial). Au même titre que n'importe quel autre parc national, le PNR serait donc écartelé entre «des sciences de gouvernance et des choix irréconciliables » (Alban $\&$ Hubert, 2013).

A La Réunion ${ }^{4}$, ces lignes de tension sont cependant renforcées par un contexte post-colonial induisant de multiples rapports de domination entre,

4 La Réunion, ancienne colonie française, est une île de l'Océan Indien, devenue département et région d'outre-Mer en 1946, un peu moins de 100 ans après l'abolition de l'esclavage sur l'île (en 1948). 
d'une part les représentants étatiques, héritiers malgré eux d'un système esclavagiste et colonial, et d'autre part les populations locales dont la mémoire collective aurait été privée d'une réflexion sur ce passé trouble (Bancel et al., 2006; Mbembe et al., 2010; Vergès, 2005; Cottias, 2006).

Nous verrons que l'outil gestionnaire EPN aux tensions multiples voire «irréconciliables» (Alban et Hubert, 2013) lorsqu'il est adopté en contexte postcolonial favorise l'émergence de sentiments d'injustice parmi les populations locales.

\section{Méthode}

Les résultats présentés ici s'appuient sur une enquête ethnographique (entretien et observation) menée durant l'année 2013 autour du projet PEIRun. Ce projet a pour objet de réaliser des « Plantations d'Espèces Indigènes (PEI) caractéristiques d'une zone géographique, pour une utilisation durable par la population et les projets d'aménagement de proximité $\gg{ }^{5}$. L'objectif conservationniste consiste à prévenir le risque d'invasion biologique en limitant le plus possible les départs d'envahissement aux frontières du cœur de parc. Il s'agit donc d'identifier un site envahi, de le défricher (l'espèce invasive ayant la particularité d'uniformiser le couvert végétal en une friche souvent épineuse) et de le replanter en espèces végétales indigènes (la manipulation des espèces endémiques étant uniquement réservée aux experts conservateurs). Après avoir choisi une forêt dont le potentiel d'envahissement constituait un risque potentiel pour les forêts du cœur de parc, les porteurs du projet doivent donc sélectionner une association volontaire pour mener cette gestion forestière en autonomie. Pour construire les connaissances utiles à l'animation de cette dimension participative, les porteurs du projet nous ont sollicité, même si notre rôle n'a pas été formulé de cette manière. Un rapport scientifique a donc été rendu, celui-ci formalise les connaissances sur la population étudiée, à savoir les riverains des forêts réunionnaises ciblées et les associatifs ${ }^{6}$ savamment sélectionnés pour conduire ces projets.

Nous avions toutefois pour ambition l'analyse de la dimension participative développée par l'ensemble des acteurs, ce qui impliquait d'inclure au recueil de données initial d'autres données concernant les porteurs du projet. Nous avons mené de nombreux entretiens avec les associatifs, assisté à des réunions, à certains conseils d'administration de ces associations, observé sur le terrain les échanges entre porteurs du projet, bénévoles et salariés de l'association lors de la mise en œuvre du projet mais nous avons également, avec la permission des porteurs de projet, enregistré toutes les réunions d'élaboration de la convention entre les porteurs et le chercheur sociologue, et tous les échanges ultérieurs entre ces deux parties (Claeys \& Thiann-Bo, 2015). Nous avons ainsi porté une égale attention à tous les types d'acteurs impliqués dans ce projet. Nous utiliserons pour des raisons d'anonymisation les termes « porteurs (de projet) » pour désigner indifféremment tous les acteurs institutionnels en charge du portage de ce projet, le terme « population » pour désigner les riverains de la forêt cible du projet et le qualificatif « associatif » pour désigner les personnes bénévoles ou salariées de cette association, même si une telle homogénéisation des catégories peut

\footnotetext{
5 Tiré de la convention entre le Parc National de La Réunion et le laboratoire IRISSE qui a réalisé la présente étude.

${ }^{6}$ Pour des raisons évidentes d'anonymisation du corpus enquêté, nous ne citerons aucune personne, aucune association ni aucun lieu pouvant permettre de reconnaître formellement le corpus des enquêtés.
} 
paraître réductrice. Dans ce récit, interviendront d'autres acteurs, associatifs ou institutionnels, partenaires des actions mises en œuvre, comme des personnels de l'ONF, acteur institutionnel gestionnaire historique des forêts domaniales.

\section{Analyse}

\subsection{La participation et l'empowerment ${ }^{7}$ comme dimensions fondatrices du projet PEIRun}

« Péï », en créole réunionnais sert à qualifier ce qui est produit en local, par extension, il désigne 1'individu autochtone («un moun péï»), à l'inverse de ce qui vient de « déor» (dehors). Prononcé à la créole, le nom de ce projet sonne comme un jeu de mots, rappel des objectifs de justice à rendre aux autochtones. Bien avant même sa mise en œuvre sur le terrain, le projet est déjà le lieu de stratégie. Il possède une logique patrimoniale reposant sur une croyance forte : il existerait une culture locale de la nature qui serait menacée et qu'il devient urgent de conserver. « il faut rendre la nature à ces habitants... eux, ils ont cette culture-là... de prendre soin de la nature » (Albert, porteur du projet). Cette hypervalorisation de la tradition semble répondre aux objectifs de conservation de la culture imposée par la loi de $2006^{8}$. Pourtant, elle fonctionne surtout comme un puissant levier de justice environnementale. En considérant comme une menace l'oubli qui pèse sur ces pratiques de la nature, les populations autochtones sont jugées spoliées de leur culture. Plus les discours font état de ces injustices, plus les porteurs de projet ont tendance à répéter, comme un leitmotiv « mais dans le tan lontan (avant) les gens savaient faire... » servant à réhabiliter un savoir ancestral et autochtone en perdition. Patrimonialiser est ainsi l'objectif de la participation citoyenne recherchée à travers la mise en œuvre du projet : il s'agit pour les porteurs de faire comprendre aux populations locales que le bien commun (les espèces endémiques) est menacé et de faire des espèces exotiques envahissantes, l'ennemi commun.

Le projet cherche à impulser un élan citoyen, et en mettant l'accent sur le riverain, cherche à construire une " population concernée » (Claeys-Mekdade, 2001). La croyance en une tradition vertueuse fait apparaître l'autochtone comme l'acteur central à solliciter. La participation « juste et équitable »selon les porteurs de projet devrait toucher d'abord l'autochtone.

Sur le papier, cette fabrique d'une population concernée par le projet ne révèle aucune remise en question du système de la conservation, mais nous nous apercevons vite que les discours off, bien au contraire le fustigent.

\begin{abstract}
« mais moi je te dis (parlant à un associatif), bien sûr que j'ai un pied de goyavier ${ }^{9}$ dans ma cour (jardin), j'adore ça moi je suis créole, mais je fais attention qu'il chape pas dehors, j'essaye de le gérer dans ma cour, c'est ça que j'aimerais expliquer à tout le monde, tu vois, que des choses comme ça c'est mieux de cultiver chez soi, d'aller cueillir dans un verger que directement dans le parc... » (René).
\end{abstract}

\footnotetext{
7 Ce cadrage théorique s'appuie pour partie sur le questionnement développé par le collectif de chercheurs agrégé autour du projet EFFIJIE (EFFort, Inéquité, Justice et Injustice Environnementales) auquel nous participons.

8 «Cette (loi) s'inscrit dans un double mouvement de reconnaissance de l'autochtonie (UICN et al., 1996; Roué, 2009) et de l'impératif participatif dans le champ de l'action publique » (Deldrève \& Candau, 2014, p. 260).

9 Espèce exotique envahissante faisant l'objet d'une cueillette traditionnelle et ayant été au centre d'une polémique à la création du parc.
} 
René réalise une double critique récurrente chez les porteurs de projet. D'abord, il se déclare autochtone, ce qui lui sert à s'afficher comme légitime, lorsque le statut de personnel du PNR stigmatise. Puis il s'affirme comme émancipé d'un savoir scientifique et de son application gestionnaire. René ne peut remettre complètement en question la dichotomie, espèce envahissante/endémique qui préside au système de conservation à La Réunion. Il ne peut donc prôner une culture du goyavier à l'échelle régionale. Un tel discours l'amènerait à renier son statut de scientifique et ses valeurs écologiques. Mais en revêtant son habit d'autochtone, il met à l'index une gestion « déraisonnable » qui souhaite conserver à tout prix, au mépris des pratiques culturelles locales. Selon lui, une «bonne » gestion environnementale se doit de respecter les goûts culturels même pour cette espèce envahissante. La gestion «raisonnée d'une peste végétale » comme le goyavier consisterait ainsi à la limiter aux consommations individuelles et à éduquer la population aux bons usages des espèces exotiques. Le projet n'a ainsi cessé de répéter son objectif : constituer un « guide des bonnes pratiques ».

L'autochtonie, facteur de légitimité pour les porteurs de projets, donne du crédit à la critique radicale du système de la conservation environnementale. Cette critique s'accompagne également d'une volonté d'agir. Et pour cela, les porteurs du projet tentent d'augmenter le plus possible leur pouvoir d'action, qu'ils considèrent comme limité dans le cadre strictement professionnel.

Cette capacité d'agir se révèle notamment dans le choix des associations qui ne s'effectue pas au hasard. La procédure de sélection attentive s'appuie à la fois sur des compétences relationnelles (développer un partenariat suivi avec l'association) et des compétences plus personnelles. Mais pris entre une tentative de conciliation d'une démarche démocratique et une démarche conservationniste, les porteurs du projet seront forcés d'employer la ruse. Le principal obstacle à une démocratie participative, selon eux, réside en effet dans la sélection d'intermédiaires légitimes. C'est par la mobilisation des réseaux privés que les porteurs œuvrent pour sélectionner un partenaire « fiable ». Le projet procède d'une forme de militantisme rusé. Ainsi, lorsque je demande à René les raisons du choix de l'association $\mathrm{ZZ}$ sur la commune $\mathrm{XX}$, il me répond :

« ben tu sais, c'est grâce à Paul (autre porteur du projet), il connaît bien Marcel (un associatif). Il est élu à la commune XX pour s'occuper de XX ... En fait c'est son cousin ${ }^{10}$ (rires). (Et) depuis longtemps il (Marcel) a un projet de réhabilitation d'un sentier pour commémorer l'esclavage. Marcel cherchait depuis longtemps à concrétiser ça, là c'est l'occasion ».

On voit ainsi que les raisons qui président au choix de l'association n'ont rien à voir avec la destination initiale du projet. Bien au contraire, puisqu'il faudra masquer sur le papier le grand écart entre la vocation du projet et la raison sociale de l'association. René me dira par la suite qu'avoir de telles relations avec les membres de l'association leur permettra d'asseoir une relation de confiance et un meilleur contrôle sur le déroulement du projet. Paul sera l'unique intermédiaire du PNR, ce qui lui permettra de rendre moins visible sa gênante institution. Marcel est quelqu'un de confiance, élu qui plus est, ce qui asseoit sa légitimité représentative (démocratique) et avec qui il sera facile de « causer des vraies intentions du projet » (René). La

10 Cette relation ne sera pas avérée sur le terrain. 
visée commémorative de l'association fait ici figure d'argument d'autorité (en faveur de l'autochtonie) et vient ouvrir un débat sur l'injustice sociale à La Réunion. Il s'agit d'éviter que le projet n'échappe à sa visée réparatrice : remettre la nature aux mains de ceux qui la méritent, les autochtones.

Choisir la «bonne » association (au sens de légitime) n'est cependant pas suffisant à leurs yeux, encore faut-il laisser la plus grande marge de manœuvre aux associatifs. Les porteurs du projet cherchent donc à effacer très vite « la patte » du parc. Il ne s'agit pas seulement d'ôter un stigmate (le PNR ayant la réputation d'être un acteur illégitime) ni de manipuler des opinions (en cachant l'interventionnisme gestionnaire). Pour eux, le rôle du parc doit se limiter à un soutien discret voire à un alibi, par exemple en servant d'intermédiaire pour appuyer les demandes de préemption des terrains ${ }^{11}$ (pour y installer les pépinières) ou en faisant bénéficier de son réseau de partenaires institutionnels. En affichant la possibilité pour les associatifs de limiter le rôle du parc à celui de pourvoyeur de fonds, les porteurs entendent lancer une impulsion pour démarrer le projet puis effacer aussitôt toute trace d'initiative institutionnelle.

On voit là s'opérer un premier glissement, entre les objectifs lisses du projet initial (doublement conservationnistes) et ceux qui sont vantés sur le terrain par ses opérateurs. Ici le projet témoigne d'une volonté réparatrice, émancipée de l'organe gestionnaire EPN, considéré comme incapable de porter à lui seul une gestion environnementale trop lourde.
« Dans un monde idéal, oui, il faudrait éradiquer toutes ces pestes, en interdisant aux jardineries de les importer, en contrôlant les cargaisons qui arrivent au Port, en contrôlant les animaleries... mais tout faire peser sur les gens c'est... les rendre responsables de tous les maux de la terre, c'est pas loyal, c'est toujours les petits qui trinquent... »

Une autre fois, Hubert m'explique comment Sarah a été élue au conseil d'administration de l'association BB, partenaire du projet dans la commune $\mathrm{CC}$ :

« ça fait longtemps que c'était prévu en fait, mais on attendait que le projet soit plus avancé. Sinon ça faisait tâche dans la convention, Sarah personnel du PNR et membre de l'association, disons un délit d'initié quoi... (rires)»

Hubert semble fier de «s sa magouille » comme il dit. Pour autant, cette comparaison ne dévalorise pas son action. En la faisant passer pour presque mafieuse, René désigne comme un méfait ce qu'il considère comme la réparation d'une inégalité de participation citoyenne. Par ce procédé rhétorique, notre interlocuteur ne se positionne plus comme un personnel du PNR mais comme un citoyen spolié de son droit à participer.

On voit s'opérer le deuxième glissement : du projet initial qui consistait à seulement « faire participer », le projet final sert également à ses porteurs à « se faire participer ».

Nous sommes loin du comportement désabusé $^{12}$ des personnels décrit par Alban et Hubert

\footnotetext{
11 Trouver un terrain pour y implanter les pépinières fut également une activité chronophage. En effet, une fois sélectionné, encore fallait-il pouvoir l'occuper dans la légalité, ce qui signifie avoir correctement identifié le propriétaire légal de l'emplacement choisi, et en même temps identifié la réglementation qui s'applique en fonction des particularités et enfin avoir opté pour un mode d'occupation du sol (location avec défraiement, sans défraiement, emprunt ou dation d'un terrain communal...).

12 «Les personnels de l'EPN ont eu tendance à développer des comportements désabusés et de repli sur eux-mêmes face à la remise en cause des méthodes de travail amenée par les chartes. » (Alban \& Hubert, 2013, p. 19).
} 
(Alban \& Hubert, 2013, p.19). Au lieu de repli sur soi, ces derniers mobilisent les sentiments d'injustice ressentis au quotidien dans leur profession pour réaliser un projet " réparateur ». Certes, la souffrance au travail est un leitmotiv dans les rencontres avec les personnels: ils ne se sentent en effet ni reconnus ni récompensés pour leur mérite ou la pénibilité de leurs tâches. Ils formulent également un profond désaccord envers un travail sur le terrain producteur d'inégalités environnementales. Mais leurs discours comme leurs pratiques cherchent à rétablir une forme de justice et à contrecarrer une hypernormativité institutionnelle.

Certains auteurs désignent les processus d'innovation sociale développés par des citoyens en matière de justice environnementale par le concept d'empowerment. L'empowerment désigne à la fois une stratégie et une dynamique de transformation sociale (Bacqué \& Biewener, 2013). Ce terme fait référence tant au pouvoir (power) («pouvoir sur », « pouvoir de » et « pouvoir avec » (Bacqué $\&$ Biewener, 2013, p. 145) qu'à la participation au gouvernement citoyen. "L'empowerment vise à renverser les rapports classiques de domination entre l'Etat et la société civile par le biais de transfert de ressources politiques et de capacité d'organisation » (Jouve, 2006, p. 5). L'empowerment est également synonyme d'émancipation dans la mesure où renverser cette domination, quelles que soient les formes que revêt cette dernière, mêle justice comme reconnaissance et justice distributive (Boltanski et al., 2014, p. 74).

Les pratiques et les discours des porteurs de projet PEIRun semblent relever d'un empowerment radical (Bacqué \& Biewener, 2013). Pour faire face à une inégalité environnementale, ils vont d'une part exprimer une critique radicale (au sens étymologique du terme, à la racine) du système conservationniste et $\mathrm{d}$ 'autre part mettre en œuvre des stratégies émancipatrices. Les porteurs dénoncent d'abord ce qu'ils perçoivent comme une injustice : à leurs yeux, une partie de la population est dépossédée de ses espaces et de ses ressources et peine à participer sous la contrainte. Ils protestent contre l'absence de prise en compte des populations locales qui ne seraient pas associées à la patrimonialisation des espaces naturels qu'on souhaite pourtant les voir protéger. Conscients de posséder un arsenal juridique « juste», ils sont pourtant frustrés des « incapacités » gestionnaires sur le terrain. En effet, la loi de 2006 sur la conservation des patrimoines culturels dans les parcs nationaux souligne la nécessité de la mise en œuvre de la participation citoyenne (par la signature des chartes notamment). Mais cette exigence de la participation citoyenne ne va pas forcément de pair avec une justice procédurale qui donnerait de manière équitable la parole à tous (Deldrève \& Candau, 2014). Avec force ruse et critique, les porteurs du projet s'insurgent contre la «capacité limitée pour les populations autochtones à faire valoir leur point de vue face à des acteurs nationaux et internationaux bien organisés qui ont déjà établi des cadres de participation 》 (Lapointe \& Gagnon, 2011, p. 154). Leur grande maitrise du cadrage national et international leur sert alors à contourner certains effets du règlement pour mieux mettre en application le vou pieu de participation citoyenne. Sur le papier, l'enjeu principal est de susciter l'auto-émancipation des populations cibles du projet. Les populations sont incitées à s'approprier les pépinières qu'elles seraient en mesure de gérer progressivement de manière autonome. Dans la pratique, il s'agit d'effacer la présence du parc pour mieux permettre, selon eux, aux populations locales de s'exprimer. Les porteurs, en robin-desbois, semblent ainsi répondre à l'invitation lancée par les chercheurs. 
"Il est impératif que les EPN résistent à cette tentation traditionnelle, qui les poussent à défendre des intérêts qu'ils pensent généraux et nationaux alors qu'ils ne sont que l'expression d'une minorité scientifique et associative, détentrice d'une vision catastrophiste et conservatrice de la nature » (Alban \& Hubert, 2013, p. 30).

Présentons maintenant les autres acteurs du projet, les populations riveraines des sites forestiers, et regardons dans quelles mesures ils parviennent ou non à y participer. Nous analyserons deux formes de participation, chacune prise dans des contraintes idoines. Pour évoquer les tensions sur le terrain entre population et gestionnaires, la plupart des auteurs parle de conflits entre les intérêts portés par les acteurs (Robert, 1998, Babou, 2015). Nous tenterons de montrer qu'ici, ce clivage procède également d'un décalage entre les éthiques développées par ces deux catégories d'acteurs quant aux bons usages de la forêt.

\subsection{Domination et émancipation de la population}

Est-ce bien utile de préciser que souvent, les éthiques ordinaires ${ }^{13}$ sont méconnues et stigmatisées? Pour ce qui concerne les usages de la forêt à La Réunion, la légitimité des institutions renvoie à l'illégitimité de ceux qui tombent sous son règlement. « Braconnage » ou cueillette et de manière générale, tous les prélèvements effectués par la population entrent en concurrence directe avec les activités de gestion forestière de l'ONF, ges- tionnaire historique des forêts de l'espace départemento-domanial réunionnais (Robert, 1998). Dans l'aire d'adhésion, l'ONF demeure, contrairement à ce qui prévaut pour les habitants du cœur de parc ${ }^{14}$, le principal concurrent des usages populaires. Ces derniers restent généralement compris dans une dichotomie : soit ils sont stigmatisés comme des actes brutaux de concurrents illégaux (le « braconnage radical » venant tuer les espèces convoitées) soit ils sont hypervalorisés car attestant d'une culture remarquable des espaces de proximité (Robert, 1998, p. 502).

Or, en tant qu'objet social, la forêt n'échappe pas aux déterminismes sociaux et aux effets de mode que subit toute société humaine (Kalaora, 1993). Du reste, la forêt semble ne pas pouvoir se penser en dehors d'une perspective gestionnaire (Arnould, P. in Veyret, 2007, p. 17) : l'espace forestier est géré à des fins sociales ou naturalistes, aménagé pour ses éventuels usagers. L'éventail des usages et des valeurs qui leur sont associés a largement été démontré. Pour autant, la méconnaissance de ces travaux fondateurs fait perdurer pour le commun des gestionnaires la stigmatisation des populations à travers la généralisation des actes particuliers de vandalisme à tous les usages de la forêt.

Or, les mondes qui cohabitent dans et autour des forêts étudiées possèdent chacun leur éthique et énoncent un certain nombre de règles fondamentales. Nous évoquerons ici deux mondes dont les acteurs expriment un fort sentiment d'injustice envers les gestionnaires de «leur» forêt.

\footnotetext{
13 Nous entendons par là les éthiques développées par les populations ordinaires, au sens où ces dernières n'occupent pas une position de gestion institutionnelle sur le terrain étudié.

14 Igor Babou décrit en effet que ce rapport est différent pour les Mafatais, habitants du cœur de parc dans la mesure où l'ONF est un fort pourvoyeur d'emploi.
} 


\subsubsection{Ethique et stratégies d'émancipation des riverains}

Le monde des zabitans est le premier qui occupera cette démonstration. La graphie de «zabitans » n'est pas une coquetterie. Nous avons voulu marquer ici la référence à «l'habitation », terme créole désignant la case (lieu physique où réside la famille) et les cultures vivrières qui lui étaient associées du temps de la colonisation. La forêt dans cet univers symbolique est un lieu de vie et de culture agricole. Elle est aussi l'extension de la « cour» (jardin, en créole), terrain de jeu propice à la découverte et à la transmission intergénérationnelle du rapport à la nature. Ce lieu, investi au quotidien, possède bon nombre de fonctions : il nourrit, protège, est terrain de jeu, d'éducation et de transmission culturelle. Le sentiment d'injustice exprimé par ces zabitans procède dès lors d'un sentiment de propriété frustré par les multiples gestionnaires qui « règnent » désormais sur ce territoire. Ces personnes se considèrent comme spoliées d'un droit de propriété légitime qu'ils avaient acquis par la coutume et la force de l'habitude. L'entrée dans un monde moderne - où le capitalisme est posé en fautif de l'expropriation injuste de ses habitants historiques- indique un avant/après caractéristique.

Une forte attribution causale concourt à l'expression de ce sentiment de spoliation : l'entrée dans la modernité de la départementalisation (en 1948) aurait introduit des logiques productivistes sur le territoire familier naguère géré comme des exploitations familiales. De nombreux récits racontent comment une grande partie de la forêt aurait été cédée ou vendue à des particuliers qui ont alors pratiqué une agriculture intensive en lieu et place des agricultures vivrières. On retrouve ici la mise en accusation d'une société de plantation où les cultures nourricières étaient systématiquement évacuées au profit d'une monoculture intensive (Benoist, 1968). Du coup, la perception de la domination du gestionnaire resté sur le territoire s'en trouve renforcée: l'ONF est raconté comme le grand vainqueur d'une lutte spatiale en ayant obtenu les faveurs de l'Etat. L'injustice perçue possède ainsi une histoire qui déroule la manière dont ce sentiment de propriété et de légitimité a été bafoué. La forêt des zabitans, tendue entre un « avant » et un « après » la déprise anthropique, est systématiquement dépeinte en référence à la présence humaine. L'humain est une pièce maîtresse dans la nature : sans lui pas d'harmonie possible.

Le récit de cette histoire des injustices qui se répètent depuis la colonisation révèle également une grammaire de l'action en forêt. Le terme d'habitation prend ici son sens littéral: les règles qui régissent la gestion du territoire forestier sont des règles domestiques. L'analogie avec la maison permet de retranscrire l'exercice de style auquel se livrent les zabitans pour décrire leur forêt : dans ce monde familier, les règles servent à la bonne tenue domestique. Trois règles fondamentales doivent ici être traduites pour comprendre ce sentiment d'injustice.

La première de ces règles est celle de l'abondance. De la même manière qu'on ne vide pas le garde-manger, pour garantir l'abondance, on ne pille pas les ressources, on les exploite sur le long terme s'exerçant parfois au-delà des limites temporelles de la vie humaine (on préserve pour soi et sa descendance). Cette règle de l'abondance indique les « bonnes manières » de prélever en forêt. Dans les discours, l'énonciation de cette règle est très vite associée à une polémique sur le braconnage. A la stigmatisation perçue du braconnage par les gestionnaires, les zabitans répondent par la distinction fondamentale entre bon et mauvais braconnier. 
La pratique du bon braconnier est systématiquement justifiée, voire excusée, et celle du mauvais condamnée sans détour.

En créole, braconner n'a pas le même sens qu'en français. Il va rimer avec maronner et signifier une forme de résistance à la domination vécue sur un territoire. Ainsi, un bon braconnèr doit prélever selon des codes. Le premier consiste à assurer l'abondance des ressources, dont il ne faut tirer de profit autre que domestique. Cette polémique est un bon moyen de saisir la grammaire du prélèvement en forêt.

Quand le braconnage est justifié, deux tendances se détachent : soit il est glorifié comme une pratique de résistance à la domination gestionnaire, soit il est l'objet d'une mansuétude à l'égard de la nécessité qui le préside. Le bon braconnage est supposé être une activité nourricière voire le moyen de pallier une malbouffe généralisée (on va prélever dans la forêt de quoi se nourrir « mieux » que dans les hypermarchés). Cette pratique viserait à rétablir une forme de justice : dans un monde globalisé où l'offre alimentaire est uniformisée, aller chercher sa nourriture par soi-même dans la forêt profite doublement. D'abord, il permet de mieux manger (le chasseur marron est sûr de la provenance de ce qu'il prélève) ; il permet aussi de résister aux tentatives des « gros blancs ${ }^{15}$ nous faire manger du lait caillé » (un enquêté fait ici référence au lait en poudre importé avant d'être reconstitué dans les usines et des rumeurs sur les « os broyés » qui composent la poudre de lait). La présentation du braconnage comme la pratique du pauvre ${ }^{16}$ finit de l'excuser complètement. Braconner par nécessité est toujours excusé par les enquêtés s'il permet de nourrir directement; la marchandisation du fruit de sa chasse est toujours condamnée dans les entretiens.

La règle d'abondance révèle la fonction principale de la forêt dans le monde des zabitans : un garde-manger dont il faut maintenir le niveau de remplissage. Tout comme pour un garde-manger, il convient aussi de s'assurer de son entretien.

La deuxième règle concerne donc la conservation en ordre de l'espace : la forêt devient un paysage où la négligence se remarque très vite. Cette règle concourt à construire l'invasion biologique comme un risque pour l'ordre domestique et relève d'une forme ordinaire de la conservation.

«I laisse pas la friche mont dessus komsa, ou laissré ou ? non, marmay koméla i gain pu défricher ${ }^{17}$ » (Raymond, 75 ans)

Cette règle d'ordre et d'harmonie se déroule, elle aussi, selon un avant et un après. Avant, la forêt est racontée comme bien entretenue grâce aux exploitations familiales. Les récits basculent dans « l'après » quand l'habitant légitime est exclu. La forêt est alors envahie, la vigne maronne (Rubus alceifolius) recouvrant les vestiges de la présence humaine et uniformisant ce qui naguère était profusion d'espèces utiles. L'invasion biologique rappelle alors l'injustice d'une déprise agricole et de la disparition de l'abondance. «On nous a chassé de là et regardez dans quel état tousa i lé, on nous

\footnotetext{
15 Les héritiers des colons d'hier ou encore les Zoreils ou métropolitains.

16 La Réunion compte 29 \% de chômage (soit plus de 100000 chômeurs) selon l'INSEE (enquête emploi 2013), « Avec 343000 personnes vivant en dessous du seuil de pauvreté monétaire, La Réunion est un département hors norme » (Indicateurs sociaux départementaux 2013, INSEE et Conseil Régional de La Réunion).

17 « On ne laisse pas le désordre s'accumuler : on défriche, on entretient »
} 
a chassé pour ne rien mettre à la place »... ou pour détourner la vocation naturelle de protection et d'abondance de la forêt pour ses habitants. Certains enquêtés font même un parallèle avec d'autres formes d'injustice comme celles qui entourent l'inapplication du droit au logement : les riverains actuels se racontent comme des autochtones soudainement déclarés « squatters » et dégagés d'un logement qui devenu vacant sera finalement laissé à l'abandon.

Pour les zabitans, la forêt représente un vaste garde-manger déserté par l'humain, où le désordre s'est installé et où la règle de l'entretien le plus minimal n'a plus été assurée. La $3^{\text {ème }}$ règle garantit la transmission et le maintien de cette conservation ordinaire.

La forêt est autant un lieu de transmission des techniques de piégeage, de chasse que de culture de la débrouillardise marronne mais elle est surtout le lieu d'exercice de la transmission de ces connaissances indispensables au maintien de l'abondance qui est assurée. Or la forêt envahie montre le joug d'une réglementation exogène qui freine cette libre transmission ; ce qui ne veut pas dire qu'elle n'a plus lieu, mais qu'elle a lieu ex situ, sans support physique, sans espace d'apprentissage grandeur nature. L'injustice naît aussi de l'impossibilité de perpétuer correctement cette règle de la transmission investie comme une nécessité.

Mais en transmettant cet arsenal de pratiques, le zabitan communique également un point de vue sur la «Loi » (la loi en créole signifiant celui qui a l'autorité). Braconner est un défi: il s'agit alors de ne pas se faire attraper par la «Loi». Assimilé à l'exécutant de cette loi injuste, le gestionnaire forestier ou la police de la nature semble être là uni- quement pour sanctionner (dans les zones protégées notamment) ; braconner sous son nez c'est braver l'interdit avec toutes les récompenses symboliques que cela génère. La bravade est rendue d'autant plus juste que la « loi » est perçue comme arbitraire: « La forêt sa lé a nou » ( « la forêt est à nous »)

Ces zabitans voient dans les gestionnaires tout particulièrement 1'ONF cristallisant tous les griefs - une domination étatique. Face au déni ou à la stigmatisation de leur propre logique de conservation du milieu, ils ne sont pas en mesure de se saisir de la logique conservationniste du projet. Ce dernier est alors considéré comme énième prétexte assimilationniste d'une domination zoreille sur des usages créoles dépréciés. La dichotomie zoreil $18 /$ créole devient alors très visible dans les discours. Participer à un projet présenté comme protecteur d'une ressource jugée détruite (car en friche) à cause des gestionnaires expropriateurs qui apparaît donc hors de propos. Les attributions causales sont d'ailleurs discordantes : à celle du projet qui énonce « il faut replanter pour protéger le patrimoine forestier » (registre normatif) les zabitans vont rétorquer « allez-y vous, c'est vous qui avez tout détruit » (registre accusateur de la faute) ou « pourquoi votre logique plutôt que la nôtre » (registre de la légitimité).

Le sentiment d'injustice des zabitans naît de deux principaux griefs. Il émerge d'abord dans l'expression d'un quotidien contrarié par les lois et les règlements exogènes qui s'appliquent sur ces territoires. Puis il semble se répéter à travers l'histoire. Le prélèvement naguère contraint par les grands propriétaires terriens l'est désormais ,par des gestionnaires, jugés d'autant plus illégitimes qu'ils viennent du continent. L'injustice sociale a laissé

18 Métropolitain, en créole, sert à désigner les individus venant de la France continentale (par opposition à la France d'Outre-Mer). 
place à, ou plutôt double, l'injustice environnementale. La frustration naît de la répétition d'une injustice qui se perpétue comme lorsque les zabitans évoquent l'abandon forcé du territoire. Dans ce jeu du récit, la constitution du Parc National, avec ses règles qui imposent encore des contraintes sur les terres, est vécue comme une forme d'impérialisme vert : les gestionnaires, comme les anciens colons imposent leur loi au colonisé.

Même en l'absence de conflit ouvert entre gestionnaires et zabitans, il est possible de considérer les pratiques des seconds comme des actes de résistance aux premiers. Se sentant traités injustement, les zabitans présentent en effet braconnage et tisanerie ${ }^{19}$ comme leur permettant de prendre un avantage symbolique sur une domination postcoloniale. La forêt « envahie » pour le regard gestionnaire, devient une friche forestière témoin de cette domination et du déclassement des autochtones qui se croyaient les propriétaires légitimes de la forêt. Leur engagement dans un processus participatif, tel que le projet PEIRun, se heurte à l'éthique ordinaire qu'ils développent au quotidien, aux antipodes de celle motivant le projet conservationniste ou gestionnaire.

\subsubsection{Le monde de la conservation ordinaire}

Le monde de la conservation ordinaire se compose de riverains que nous appellerons « gardiens », eu égard aux prérogatives octroyées sur le territoire forestier. Tous ne possédaient pas les mêmes statuts. Pour la plupart, ils sont au moment de l'entretien ou ont été employés par l'association choisie pour mener le projet. Ils étaient « emplois verts » qualifiés ou non sur la durée du projet, ou encadrants avec plus ou moins de responsabilités. Les associations sélectionnées étaient alors en mesure d'obtenir des financements pour l'entretien des espaces forestiers et ne fonctionnaient pas uniquement sur du bénévolat. Certaines associations comptaient toutefois plus de bénévoles actifs ; ces derniers avaient alors l'avantage de posséder les territoires forestiers choisis pour la mise en œuvre du projet. Ne pouvant accueillir des emplois verts sur leur propriété, ils faisaient alors appel à des formes de solidarités (associatives, familiales ou de voisinage) pour les aider au défrichage des terrains.

Le monde des gardiens échappe donc à l'unique prévalence des variables sociologiques classiques (il ne réunit pas seulement des détenteurs d'un même capital socio-culturel) et se construit pour partie selon une théorie de l'action sur l'espace. Ces individus forment un monde parce qu'ils ont la charge, à un moment donné, de le défricher, de le replanter ou de l'entretenir, voire d'en repenser la destination. Bien que riverains d'un territoire donné, tous ne se connaissent pas forcément. Le mélange des générations aidant ${ }^{20}$, les « zistwar lontan » (histoires d'avant) sont partagées durant les pauses. La pénibilité du travail de défrichage vient alors ajouter du liant aux vécus : le sentiment d'appartenir à une communauté d'épreuve favorise la transmission d'une histoire du lieu, se construisant peu à peu comme une histoire commune, au fil des récits et de l'identification des tiers acteurs de cette histoire (les auditeurs reconnaissant dans les personnages des récits des membres de leur famille

\footnotetext{
19 Activité liée à l'art de faire des tisanes, de la cueillette des plantes médicinales à la constitution des remèdes.

20 Sur une des communes enquêtées, la politique de création des emplois verts est pourvoyeuse d'emplois pour des catégories de population qui en sont le plus éloignées : jeunes et moins jeunes se retrouvent alors collègues durant le temps souvent très court du contrat de travail accordé.
} 
ou des proches voisins). Les longues marches de reconnaissance dans les forêts sont aussi l'occasion de partager ces zistwar et d'éprouver les liens, jusqu'alors inconnus, qui unissent les acteurs en présence. La forêt devient support physique de rapprochement des individus, de leurs histoires autant que de leurs valeurs.

Ce monde s'organise autour de deux principaux pôles selon les logiques de patrimonialisation qui animent l'investissement dans le projet. Ces patrimonialisations qui consistent en la sacralisation de l'objet patrimonial ne possèdent pas toutes les mêmes temporalités. Soit la fabrique du bien commun a lieu en cours de projet : le patrimoine s'érige au fur et à mesure de la réhabilitation de la forêt qui fait redécouvrir une histoire locale d'un rapport avec la nature. Soit cette construction est antérieure au projet et les pratiques forestières sont instrumentalisées pour permettre la valorisation d'une identité locale (revivalisme du marronnage).

Les gardiens se distinguent aussi par des volontés bien distinctes de protéger la forêt même si dans les deux cas, il s'agit de réintroduire l'humain pour effacer les marques de l'abandon (la friche, l'invasion biologique). La première cherche à sanctuariser les lieux en y excluant les usages jugés illégitimes. Les plus anciens gardiens, ceux qui ont connu la forêt avant les premières opérations de défrichage, fustigent par exemple son ancienne fonction de décharge sauvage : certains riverains venant y déposer leurs déchets ménagers. La protéger revient alors à la préserver de ces « dégradations » extérieures tout en enfouissant ses « trésors » (des espèces végétales prisées). La forêt devient une extension du domaine privé à clôturer sous peine que « tout serait volé ». La seconde tend vers une muséification de la forêt: il s'agit de la protéger de l'oubli en dévoilant la collection d'espèces végétales et/ou animales resituée dans une histoire commune; le but étant de créer un haut lieu de mémoire de l'esclavage ou du marronnage. La forêt est ici davantage un lieu de sociabilité.

C'est dans la tension entre ces deux formes de patrimonialisation (entre forêt-sanctuaire et forêt-musée participatif) que la forêt révèle la mise en œuvre des déterminants sociaux. Ce sont en effet les mieux dotés socialement, ceux qui sont les mieux assurés de leur capital d'autochtonie (Rettière, 2008) qui souhaitent ouvrir la forêt à ses anciens usages perçus de sociabilité, les moins bien dotés préférant la clôturer pour en préserver la valeur. Comme pour les ouvriers de Lanester décrits par Retière, les uns se replient sur le domaine privé quand d'autres investissent les lieux collectifs (Retière, 2008, p. 143). A l'individualisation du processus de patrimonialisation des gardiens-sanctuaire, la fabrique du patrimoine des autres (les gardiens-participatifs) réplique par un haut «degré de communautarisation » (Retière, 2003, p. 130). Ces derniers expriment haut et fort un militantisme politique. Mobilisant un capital social et une identité culturelle valorisée, ces enquêtés affichent une forte imbrication des espaces de vie. Henri a 80 ans et se sert de son jardin pour conserver les espèces endémiques rares ou en danger puis il prend un malin plaisir ensuite à disséminer " ses » graines dans la forêt. Il sait bien être dans l'illégalité ${ }^{21}$ mais comme nul ne peut le prouver, il ironise devant des gestionnaires «futurs grands découvreurs » des espèces censément disparues qu'il aurait lui-même replantées. Sa science ordinaire, faite d'héritage personnel et d'une curiosité insatiable rivalise d'in-

${ }^{21}$ Seul le Conservatoire Botanique peut conserver et replanter des espèces endémiques. 
telligence avec celle des « experts et scientifiques ». Sa rébellion s'effectue autant dans sa cour que dans la forêt, mêlant espace privé et collectif et attestant de la non-séparation des champs de pratique (Retière, 2003, p. 130). Henri dispose de son capital d'autochtonie comme ressource à opposer aux experts proclamés des sciences de la nature dont se réclament les gestionnaires.

Les stratégies d'empowerment déployées par les gardiens permettent ainsi de maintenir à distance experts et gestionnaires. La remise en cause est d'abord radicale puisqu'elle dénonce les catégorisations savantes de la nature (bonne et mauvaise nature); elle s'attache ensuite à déconstruire les chaines d'attribution causale de l'envahissement des espaces naturels (la déprise agricole et non plus la trop grande pression anthropique-argument gestionnaire).

En ce sens, nos résultats rejoignent l'analyse des critiques de la catégorisation des invasions biologiques (Menozzi, 2010) mettant en avant les désaccords quant au critère de l'origine pour classer les invasives. Les jugements de valeur en jeu dans les catégorisations du vivant ont déjà amené certains auteurs à mettre en cause une « définition politique » des espèces invasives (Lodge \& Shrader-Frechette, 2003).

Armés de cette critique épistémologique, il devient possible alors pour les gardiens de comparer les instances gestionnaires à un «dépositaire d'une prétention à incarner le point de vue de la totalité (...) [clôturant ainsi] l'espace des définitions possibles de la réalité » (Boltanski et al., 2014 p. 52). Les individus souhaitent s'auto-émanciper face à la domination perçue des experts ; l'empowerment est donc radical.
Ces sentiments d'injustice procèdent d'abord d'une polémique autour des savoirs produits sur la forêt et des catégorisations savantes. Le savoir scientifique des experts est ramené à une croyance fondée sur des partis-pris politiques. Les rhétoriques avancées opèrent un glissement du biologique au social : 1'expertise savante est jugée à travers le prisme de la créolisation comme aussi « étrangère/ exotique » que les espèces qu'elles dénomment. Certaines espèces considérées nuisibles par les gestionnaires sont réhabilitées par endogénisation ${ }^{22}$ (Thiann-Bo Morel, 2012).

L'expression de l'injustice provient ensuite d'une polémique sur les bons usages et la manipulation des espèces. La catégorisation et l'identification des espèces à prohiber leur semblent être exclusivement l'affaire des experts scientifiques au détriment des usages et surtout des croyances des populations locales. Les gardiens formulent clairement cette « très grande inégalité par rapport à la règle » (Boltanski et al., 2014, p. 51) : la population locale n'aurait qu'à se conformer, en ayant été au préalable informée et (ré)éduquée aux bonnes façons de faire.

Deux visions du monde se heurtent. Même s'ils semblent s'accorder avec les experts sur la valeur du risque invasion biologique, les « gardiens » divergent sur les réponses à lui apporter, révélant alors un conflit sur l'opérationnalisation des valeurs. Ce conflit est cristallisé sur le choix des espèces légitimes à conserver dans la forêt. Le même genre de débat opère sur la manipulation des espèces endémiques dont l'usage serait exclusif des experts mais que les gardiens souhaiteraient utiliser pour faire revivre des usages ancestraux. Toutefois,

\footnotetext{
22 Lors d'une enquête quantitative réalisée en 2010 sur les espèces exotiques envahissantes, nous avons montré que les individus qui croient en l'utilité d'une espèce ont tendance à la dire autochtone ou endogène (Thiann-Bo, 2012).
} 
le conflit s'organise aussi autour de la légitimité des espèces à planter. Les gardiens posent ainsi souvent ces questions : pourquoi ne peuvent-ils pas cultiver certaines espèces envahissantes ? Pourquoi le droit de planter des espèces endémiques est-il réservé à quelques-uns?

\section{Conclusion}

\subsection{Domination et justice environnementales en contexte postcolonial}

Avant de conclure, nous souhaitons nous attarder sur la fonction argumentative de l'autochtonie. Nous avons vu que la revendication de cette dernière s'effectue à des fins différentes : elle assoit la légitimité chez les porteurs de projet et les gardiens, elle révèle les injustices chez les zabitans. L'autochtonie comme révélateur des inégalités environnementales renvoie ici au contexte postcolonial réunionnais. $\mathrm{La}$ grille de lecture du postcolonialisme est très rarement déployée pour décrire la société réunionnaise. Or, celle-ci permet de saisir les logiques à l'œuvre dans cette société en termes de rapport de domination:

«La postcolonie ne qualifie pas strictement un régime d'indépendance nationale, mais une situation où perdurent des effets du régime colonial, tout en connaissant de nouvelles expériences engendrées par le déclin des productions locales, l'entrée dans l'espace européen, la mondialisation, l'augmentation du nombre de diplômés, l'émergence de revendications de réparation historique et d'affirmation de différence culturelle. » (Vergès, 2005, p. 69)
L'emploi d'un outil gestionnaire « parc national » invite déjà à penser les rapports de force entre global et local, entre un discours universaliste et des mises en œuvre environnementales sur le terrain (Alban \& Hubert, 2013). A la fracture de l'universalisme, cet outil ajoute une autre ligne de fracture culturelle : celle de l'environnementalisme. Or, ces lignes de tensions viennent se heurter à d'autres facteurs d'inégalités sociales héritées de la société de plantation et sont aggravées par la situation post-coloniale. Elles exacerbent effectivement l'idée qu'entre "mère-trop-pôle » (Cambefort, 2001) et Réunion, le hiatus n'a pu que se creuser, les deux entités entretenant un rapport paradoxal.

Le post-colonialisme engage à la prise en compte dans l'analyse de l'effet de variables ethniques et raciales en vigueur dans ces sociétés. La réglementation de l'usage d'un espace naturel intervient dans un milieu social où le «foncier (est) fortement inégalitaire » (Vergès, 2005, p. 74) et où la demande d'accès aux aménités environnementales augmente (Robert, 1998). La gestion de ces $42 \%$ du territoire insulaire mis en réserve, procède d'une unité nationale, territoriale républicaine là où des particularismes locaux sont revendiqués, en sachant que ces particularismes sont construits de manière fragmentée « à partir de leur propre lieu et (utilisent) l'esclavage comme ressort politique » (Cottias, 2006, p. 180). De la même manière, ces revendications amènent à convoquer les identités des communautés « imaginées » comme ressort politique dans des négociations (Cottias, 2006).

Cette perspective socio-historique apparaît essentielle pour comprendre la complexité des rapports sociaux dans les DOM-TOM ${ }^{23}$. Or, le fait colonial est largement évacué en raison d'un

\footnotetext{
23 Depuis 2003, l'appellation officielle pour les territoires d'Outre-Mer est DROM-COM (Département et Région d'Outre-Mer et Collectivités d'Outre-Mer) mais le sigle DOM-TOM est resté dans l'usage courant.
} 
silence institutionnalisé autour de l'esclavage et de la colonisation ${ }^{24}$. Lorsque ces enjeux de domination postcoloniale sont malgré tout socialement saisis, ils mettent au jour des revendications mémorielles et la question de la réparation, tout du moins celle des responsabilités. Comme il y a très peu de lieux où une mémoire collective endogène pourrait se formaliser, cette dernière connaît des "phénomènes de mémoire compensatoire qui se sont emparés $d u$ vocabulaire victimaire pour exiger une repentance» (Vergès, 2005, p. 72). Notre analyse a tenté de dépasser l'exposé des discours de dénonciation en regardant dans quelles mesures ils entraînent la formation des sentiments d'injustices.

\subsection{De quelques erreurs et décalages entre} logique de projet et logique d'investissement du public ciblé

Sentiments d'injustice et désirs de plus de justice se répondent donc lors de la mise en œuvre du projet PEIRun. Retenons d'abord que la justice environnementale portée par ce projet est indissociable de l'activité des personnels impliqués. Leur positionnement militant tente de réconcilier identité professionnelle et identité personnelle. S'ils s'accordent du point de vue des valeurs avec leur institution de rattachement, l'opérationnalisation de ces valeurs entre en conflit avec des identités personnelles qui se saisissent des questions de domination postcoloniale. En dépit d'une critique radicale de la politique du parc, ce qu'ils souhaitent impulser sur le terrain est beaucoup plus progressif. Ces personnels rusent et passent les rouages administratifs les plus contraignants, ils manipulent la grammaire étatique et gestionnaire au profit d'une équité environnementale et des « vraies » valeurs (à leurs yeux) de la conservation.

A contrario, la conservation ordinaire, elle, révèle toute l'ambigüité du mot " conserver: maintenir dans son état initial ». Le point zéro de la forêt pour les riverains-zabitans est celui où leur présence dans le milieu leur permettait de subvenir à leurs besoins en conservant, de leur point de vue, et les ressources et l'espace. La perspective gestionnaire enracinée plus loin dans le temps se focalise aujourd'hui sur l'origine des espèces (endémiques contre exotique) et reste hermétique à cette vision ordinaire des choses. En outre, la critique épistémologique des gardiens éprouve la scientificité des méthodes des experts et ouvre sur d'autres opportunités de conflit. Les représentations du bon ordre à rétablir s'affrontent, constituant autant de possibilités d'écueil du projet.

Les principaux décalages sont produits par les croyances fondatrices du projet. La logique de patrimonialisation a priori des personnels du parc aboutit à une solution toute trouvée à l'injustice : le rétablissement des pratiques traditionnelles jugées vertueuses. Toutefois, cette survalorisation de la tradition s'effectue au détriment des usages vernaculaires qui se réalisent parfois dans le refus de la tradition ou de la réconciliation.

Le projet tente d'impulser une justice procédurale (Schlosberg \& Carruthers, 2010). Mais il prend le risque de rester incantatoire car s'il reconnait la légitimité des autochtones et souhaite leur donner la parole, il oublie qu'ils la prennent déjà. Une justice procédurale apparaît ainsi négligée au profit de la justice comme reconnaissance. Cette négligence est rendue moins visible par le militantisme des gar-

\footnotetext{
24 Françoise Vergès, dans son article sur l'utopie coloniale, évoque ainsi le glissement sémantique opéré par la substitution historique effectuée lors de la départementalisation de La Réunion : de colonie la Réunion est devenue département d'Outre-mer) (Vergès, 2005).
} 
diens, qui, en répondant au militantisme professionnel est l'arbre qui cache la forêt. L'empowerment radical des porteurs de projets et celui des gardiens dialoguent; mais ignorent les raisons qui amènent les zabitans (dans cette enquête) à faire perdurer les pratiques de l'ombre.

Du reste, l'affichage d'une justice sur le mode unique de la reconnaissance amène à des dérives. La stigmatisation des gestionnaires renvoyés à des catégories ethniques dominantes renforce du coup la présentation de soi comme victime minoritaire et propose moins une réconciliation qu'une forme de réparation. Or, la situation postcoloniale impose un mélange des genres. On n'a pas à faire à des entités distinctes mais mixtes enracinées dans un « mi-mi » (et non dans un « ni-ni ») (Memmi, 1957). En faisant disparaître le poids institutionnel du PNR, les porteurs du projet oublient que la réparation des injustices en contexte postcolonial passe aussi par la réconciliation entre des entités

\section{Références}

Alban, N.; Hubert, G. Le modèle des parcs nationaux à l'épreuve du territoire. VertigO - la Revue Electronique en Sciences de l'Environnement, 13(2), 2013.

Babou, I. Patrimonialisation et politiques de la nature: le parc national de La Réunion. VertigO - la Revue Electronique en Sciences de l'Environnement, 15(1), 2015.

Bancel, N.; Blanchard, P.; Vergès, F. La République coloniale. Hachette Littératures, 2006.

Baret, S.; Baider, C.; Kueffer, C.; Foxcroft, L. C.; Lagabrielle, E. Threats to Paradise? Plant invasions in Protected Areas of the Western Indian Ocean Islands. In: Foxcroft, L. C.; Pyšek, P.; Richardson, D. M.; Genovesi, P. (Eds.). Plant Invasions in Protected Areas: Patterns, Problems and Challenges. Springer, Dordrecht, 2013. p. 423-447.

Baret, S.; Lavergne, C.; Fontaine, C.; Saliman, M.; Hermann, S.; Triolo, J.; Bazil, S.; Sertier, J.-C.; Lequette, B.; contraires dont sont porteurs les héritiers métissés de la société de plantation.

Les multiples décalages (entre les éthiques de la nature et les formes de patrimonialisation) ont détourné les objectifs initiaux du projet et abouti à un objectif minimal : défricher pour limiter les départs d'invasions et replanter au fur et à mesure de l'établissement d'artefacts de consensus autour des espèces à planter.

Les résultats de cette enquête montrent que pour qu'il y ait participation, il faudrait a minima concordance des valeurs même au risque d'un conflit sur 1'opérationnalisation des valeurs. Toutefois, est-ce pour autant un échec de la participation ou bien peut-on considérer que d'autres objectifs démocratiques ont été atteints? En effet, une fois l'espace forestier réhabilité, ce dernier s'est vu occuper par d'autres usagers, se réappropriant l'espace et les espèces, signe d'un « revivalisme culturel sans patrimonialisation » (Lenclud, 1987)?

Gigord, L.; Lucas, R.; Picot, F.; Muller, S. Une méthodologie concertée pour la sauvegarde des plantes menacées de l'île de La Réunion. Revue d'Ecologie (Terre et Vie), Suppl. 11, 85-100, 2012.

Benoist, J. Types de plantations et groupes sociaux à la Martinique. Cahiers des Amériques latines, 2, 130-160, 1968.

Cambefort, J.-P. Enfances et familles à la Réunion. Paris: L'Harmattan, coll. « Logiques sociales », 2001.

Claeys-Mekdade, C. Qu'est-ce qu'une population concernée. L'exemple camarguais. Géocarrefour, 76(3), 217-223, 2001.

Clayes, C.; Sirost, O. (2010). Natures proliférantes. Introduction. Etudes Rurales, 185, 9-22, Janvier-juin 2010.

Claeys, C.; Thiann-Bo Morel, M. The contribution of sociology to biological invasions analysis: feedback from 
experiences and perspectives of research in order to overcome methodological disappointments and epistemological irritations. Revue d'Ecologie (Terre et Vie), 70(4-7), suppt 12 «Espèces invasives», 175-191, 2015.

Cottias, M. Sur l'histoire et la mémoire de l'esclavage? Cités, 25(1), 178-180, 2006.

Deldrève, V.; Candau, J. Produire des inégalités environnementales justes? Sociologie, 5(3), 2014.

Kalaora, B. Le musée vert: radiographie du loisir en forêt. Paris: L'Harmattan, 1993.

Lapointe, D.; Gagnon, C. A l'ombre des parcs: la conservation comme enjeu de justice. Justice et injustices environnementales (Presses Universitaires de Paris Ouest, p. 149-169). Paris: Blanchon et al., 2011.

Lenclud, G. La tradition n'est plus ce qu'elle était... Terrain, 9, 110-123, 1987.

Lodge, D. M.; Shrader-Frechette, K. Nonindigenous Species: Ecological Explanation, Environmental Ethics, and Public Policy. Conservation Biology, 17(1), 31-37, 2003. $<$ http://doi.org/10.1046/j.1523-1739.2003.02366.x>.

Mbembe, A.; Vergès, F.; Bernault, F.; Boubeker, A.; Bancel, N.; Blanchard, P. Ruptures Postcoloniales. Paris: La Découverte, 2010.

Menozzi, M.-J. Comment catégoriser les espèces exotiques envahissantes. Etudes rurales, 185(1), 51-66, 2010.
Memmi, A. Portrait du colonisé. Précédé de Portrait du colonisateur. Paris, Éditions Corréa, 1957.

Nussbaum, M.; Sen, A. (Éd.). The Quality of Life. Oxford: Oxford University Press, 1993.

Robert, R. La gestion et la valorisation du domaine public dans les Hauts de l'île de la Réunion (océan Indien). Annales de Géographie, 107(603), 487-507, 1998.

Schlosberg, D. Reconceiving Environmental Justice: Global Movements And Political Theories. Environmental Politics, 13(3), 517-540, 2004.

Schlosberg, D. Defining Environmental Justice: Theories, Movements, and Nature. Oxford: Oxford University Press, 2007.

Schlosberg, D.; Carruthers, D. Indigenous Struggles, Environmental Justice, and Community Capabilities. Global Environmental Politics, 10(4), 12-35, 2010.

Sen, A. L'idée de justice. Paris: Flammarion, 2010.

Thiann-Bo Morel, M. Biodiversité et invasions biologiques, ce qu'en pensent les Réunionnais. Protection de la biodiversité Outre-mer. Approches pluridisciplinaires (Presses Universitaires d'Aix-Marseille). Aoustin, Tirstan, Maisonneuve, Mathieu, Naim-Gesbert, Eric. 2012.

Vergès, F. L'Outre-Mer, une survivance de l'utopie coloniale républicaine? Cahiers Libres, 4, 67-74, 2005.

Veyret, Y. Dictionnaire de l'Environnement. Paris: A. Colin, 2007. 